\title{
Molecular surveillance of multi- and extensively drug- resistant tuberculosis transmission in the European Union from 2003 to 2011
}

\author{
J L De Beer (jessica.de.beer@rivm.nl)1 , C Ködmön², M J van der Werf², J van Ingen³, D van Soolingen ${ }^{1,3,4}$, the ECDC MDR-TB \\ molecular surveillance project participants ${ }^{5}$ \\ 1. National Tuberculosis Reference Laboratory, Laboratory for Infectious Diseases and Perinatal Screening (LIS), Centre for \\ Infectious Disease Control (CIB), National Institute for Public Health and the Environment (RIVM), Bilthoven, The Netherlands \\ 2. European Centre for Disease Prevention and Control (ECDC), Stockholm, Sweden \\ 3. Department of Medical Microbiology, Department of Medical Microbiology Nijmegen, The Netherlands \\ 4. Department of Pulmonary Diseases, Radboud University Nijmegen Medical Centre, Nijmegen, The Netherlands \\ 5. The participants are listed at the end of the article
}

Citation style for this article:

De Beer JL, Ködmön C, van der Werf MJ, van Ingen J, van Soolingen D, the ECDC MDR-TB molecular surveillance project participants. Molecular surveillance of multiand extensively drug-resistant tuberculosis transmission in the European Union from 2003 to 2011. Euro Surveill. 2014;19(11):pii=20742. Available online: http://

www.eurosurveillance.org/ViewArticle.aspx?Articleld=20742

The European Centre for Disease Prevention and Control (ECDC) initiated a project on the molecular surveillance of multi- and extensively drug-resistant tuberculosis (MDR-/XDR-TB) transmission in the European Union (EU) in the period from 2009 to 2011. In total, 2,092 variable number of tandem repeat (VNTR) patterns of MDR-/XDR-TB Mycobacterium tuberculosis isolates were collected, originating from 24 different countries in the period 2003 to 2011. Of the collected VNTR patterns, $45 \%(n=941)$ could be assigned to one of the 79 European multiple-country molecular fingerprint clusters and $50 \%$ of those $(n=470)$ belonged to one extremely large cluster caused by Beijing strains of one genotype. We conclude that international transmission of MDR-/XDR-TB plays an important role in the EU, especially in the eastern part, and is significantly related to the spread of one strain or clone of the Beijing genotype. Implementation of international cluster investigation in EU countries should reveal underlying factors of transmission, and show how TB control can be improved regarding case finding, contact tracing, infection control and treatment in order to prevent further spread of MDR-/XDR-TB in the EU.

\section{Introduction}

Molecular surveillance of multi- and extensively drugresistant tuberculosis (MDR-/XDR-TB) in the European Union (EU) on basis of IS6110 restriction fragment length polymorphism (RFLP) typing detected large molecular clusters of MDR-/XDR-TB cases across EU countries in the period 2003 to 2007 [1]. It also identified possible transmission patterns and risk factors for MDR-TB and XDR-TB, such as country of origin and infection with the Beijing genotype [2]. Following up on these findings, the European Centre for Disease Prevention and Control (ECDC) initiated a molecular surveillance project on MDR-/XDR-TB in the EU from
2009 to 2012 which was built on the existing TB network previously funded by the European Commission. This new project, carried out by the National Institute for Public Health and the Environment (RIVM) on behalf of the ECDC, aimed at achieving a higher coverage by expanding molecular typing to countries in the EU where this was not yet the practice. For this purpose, the 24-locus mycobacterial interspersed repetitive unit variable number of tandem repeat (MIRU-VNTR) typing method was selected as the main DNA fingerprinting methodology [3]. This method has become the international gold standard for typing of Mycobacterium tuberculosis isolates and offers important advantages over IS6110 RFLP typing, while its discriminatory power equals that of IS6110 [3,4]. Firstly, VNTR typing is easier to perform than RFLP typing and can be implemented more efficiently in countries that do not yet perform molecular typing. Secondly, it is based on DNA amplification, which abolishes the need for culture of M. tuberculosis and has a shorter laboratory turnaround time. Moreover, this approach uses low quantities of DNA and allows exchange of (non-viable) mycobacterial culture material by regular mail. Finally, the results of VNTR typing are in a simple format, which facilitates efficient exchange of typing information and inter-laboratory comparison. In principle, this introduces more real-time typing and rapid feedback on molecular clustering to identify newly emerging MDR-/ XDR-TB strains.

This paper describes the major findings of the ECDC/ RIVM project regarding the detection of international clusters, the molecular typing coverage of MDR-/ XDR-TB cases, the conclusions drawn from molecular analysis and recommendations for the future development of molecular surveillance of MDR-/XDR-TB in the EU. 
Culture-confirmed multi- and extensively drug-resistant tuberculosis cases reported to the TESSy system, and coverage in the molecular surveillance project, by country, 2003-2011 $(\mathrm{n}=16,858)$

\begin{tabular}{|c|c|c|c|c|c|c|c|c|c|c|c|c|}
\hline \multirow{2}{*}{$\begin{array}{l}\text { Country of } \\
\text { isolation }\end{array}$} & \multicolumn{9}{|c|}{ Year of isolation } & \multirow{2}{*}{$\begin{array}{l}\text { Total } \\
\text { reported to } \\
\text { ECDC } \\
2003^{-11}\end{array}$} & \multirow{2}{*}{$\begin{array}{c}\text { Total with } \\
\text { molecular } \\
\text { surveillance } \\
\text { data } \\
2003-11\end{array}$} & \multirow{2}{*}{ Coverage } \\
\hline & 2003 & 2004 & 2005 & 2006 & 2007 & 2008 & 2009 & 2010 & 2011 & & & \\
\hline Austria & 12 & 19 & 13 & 10 & 9 & 15 & 22 & 15 & 19 & 134 & $N R$ & NA \\
\hline Belgium & 9 & 12 & 11 & 18 & 14 & 21 & 10 & 19 & 15 & 129 & 30 & $23 \%$ \\
\hline Bulgaria & 44 & 47 & 47 & 53 & 76 & 31 & 43 & 56 & 55 & 452 & 141 & $31 \%$ \\
\hline Croatia & 8 & 3 & 7 & 3 & 3 & 4 & 7 & NR & 2 & 37 & 56 & $151 \%^{a}$ \\
\hline Cyprus & 0 & 0 & 1 & 0 & 3 & 1 & 4 & 0 & 1 & 10 & 1 & $10 \%$ \\
\hline Czech Republic & 2 & 6 & 13 & 12 & 8 & 11 & 8 & 9 & 7 & 76 & 37 & $49 \%$ \\
\hline Denmark & 0 & 0 & 5 & 3 & 2 & 0 & 2 & 2 & 3 & 17 & 15 & $88 \%$ \\
\hline Estonia & 106 & 90 & 78 & 55 & 82 & 73 & 85 & 63 & 78 & 710 & 557 & $78 \%$ \\
\hline Finland & 3 & 0 & 2 & 2 & 2 & 1 & 6 & 6 & 5 & 27 & 20 & $74 \%$ \\
\hline France & 25 & 26 & 24 & 30 & 20 & 27 & 30 & 23 & 40 & 245 & 87 & $36 \%$ \\
\hline Germany & 91 & 98 & 103 & 82 & 66 & 49 & 62 & 48 & 56 & 655 & 62 & $9 \%$ \\
\hline United Kingdom & 52 & 47 & 40 & 51 & 56 & 53 & 60 & 60 & 81 & 500 & 120 & $24 \%$ \\
\hline Greece & 22 & 16 & 12 & 13 & 14 & 0 & 14 & 0 & 5 & 96 & 48 & $50 \%$ \\
\hline Hungary & 20 & 11 & 17 & 14 & 12 & 13 & 18 & 19 & 7 & 131 & 36 & $27 \%$ \\
\hline Ireland & 1 & 2 & 3 & 4 & 7 & 2 & 1 & 2 & 3 & 25 & 18 & $72 \%$ \\
\hline Italy & 42 & 24 & 22 & 28 & 56 & 71 & 82 & 87 & 81 & 493 & 248 & $50 \%$ \\
\hline Latvia & 174 & 195 & 161 & 142 & 99 & 128 & 131 & 87 & 95 & 1,212 & 30 & $2 \%$ \\
\hline Liechtenstein & NR & NR & $N R$ & NR & NR & NR & NR & NR & NR & 0 & $\mathrm{NR}$ & NA \\
\hline Lithuania & 312 & 318 & 338 & 332 & 314 & 276 & 322 & 310 & 296 & 2,818 & 73 & $3 \%$ \\
\hline Luxembourg & 1 & 1 & 0 & 0 & 0 & 0 & 0 & 0 & 2 & 4 & NR & NA \\
\hline Norway & 3 & 4 & 3 & 3 & 3 & 4 & 8 & 8 & 4 & 40 & 28 & $70 \%$ \\
\hline Poland & 92 & 51 & 46 & 32 & 22 & 19 & 21 & 30 & 41 & 354 & NR & NA \\
\hline Portugal & 23 & 35 & 31 & 22 & 34 & 32 & 24 & 19 & 22 & 242 & NR & NA \\
\hline Romania & 585 & 810 & 849 & 673 & 673 & 792 & 624 & 502 & 530 & 6,038 & NR & NA \\
\hline Slovakia & 6 & 1 & 8 & 7 & 7 & 4 & 1 & 1 & 3 & 38 & 13 & $34 \%$ \\
\hline Slovenia & 1 & 0 & 1 & 1 & 0 & 2 & 1 & 0 & 0 & 6 & 6 & $100 \%$ \\
\hline Spain & 47 & 59 & 80 & 50 & 59 & 76 & 56 & 49 & 41 & 517 & 242 & $47 \%$ \\
\hline Sweden & 7 & 6 & 4 & 3 & 15 & 11 & 13 & 18 & 17 & 94 & 75 & $80 \%$ \\
\hline The Netherlands & 8 & 3 & 3 & 5 & 3 & 13 & 20 & 11 & 15 & 81 & 92 & $114 \%^{a}$ \\
\hline Turkey & 0 & 0 & 191 & 249 & 240 & 263 & 222 & 250 & 262 & 1,677 & 20 & $1 \%$ \\
\hline \multicolumn{10}{|l|}{ Total } & 16,858 & 2,055 & $12 \%$ \\
\hline
\end{tabular}

NA: not applicable; NR: not reported; ECDC: European Centre for Disease Prevention and Control; TESSy: The European Surveillance System at ECDC.

${ }^{\text {a }}$ More than $100 \%$ coverage is the result of incomplete culture data collection by the ECDC.

\section{Methods}

\section{Project design}

Molecular typing data of MDR-/XDR-TB cases from EU countries were collected in the period from 2009 to 2011 by the RIVM in Bilthoven, the Netherlands. Furthermore, retrospective typing of isolates collected from patients in the period from 2003 to 2008 and real-time typing of isolates collected from patients from 2009 to the end of 2011 were included. The RIVM reported clustering of MDR-/XDR-TB cases to the ECDC on a regular basis. In addition, the implementation, standardisation and quality control of VNTR typing in all participating countries was facilitated by ad hoc email contact, on-site training, by project meetings and workshops, and also by the introduction of a proficiency testing programme for VNTR typing [5]. The collection of samples did not follow a rational selection but was driven by the specific situation in the different participating countries.

\section{Participants in the project}

This molecular surveillance project was designed for all EU, European Economic Area (EEA), and EU 
candidate countries. The countries with national reference laboratories participating in the project were: Austria, Belgium, Bulgaria, Croatia, Cyprus, the Czech Republic, Denmark, Estonia, Finland, France, Germany, Greece, Hungary, Ireland, Italy, Latvia, Liechtenstein, Lithuania, Luxembourg, the Netherlands, Norway, Poland, Portugal, Romania, Slovakia, Slovenia, Spain, Sweden, Turkey and the United Kingdom.

\section{MIRU-VNTR typing}

The standard for typing $M$. tuberculosis complex strains was the method described by Supply et al. in 2006 [3]. The RIVM offered MIRU-VNTR typing to the countries that were not able to perform the technique locally or were not performing it for other reasons. Specifically, the RIVM performed VNTR typing for Cyprus, the Czech Republic, Estonia, Greece, Hungary, Latvia, Norway, Slovakia and Spain, and partial typing for Finland and Lithuania.

\section{Drug susceptibility testing}

Phenotypic drug susceptibility testing (DST) was performed by the TB reference laboratories participating in the project. All $M$. tuberculosis isolates were tested at least for resistance to the first-line antibiotics rifampicin and isoniazid, and part of the strains were also tested for resistance against second-line antibiotics such as fluoroquinolones and the injectable drugs (capreomycin and aminoglycosides), according to national guidelines for DST. All participating laboratories were members of the European Reference Laboratory Network (ERLN)-TB and had their own national accreditation.

\section{Molecular assessment of susceptibility by MTBDRplus assay}

We selected for molecular assessment strains that belonged to the largest European MDR-TB cluster, with a view of including a wide spread of country and year of isolation. For selected strains, the GenoType MTBDRplus reverse line blot method (HAIN Lifescience, Nehren, Germany) [6] was applied to detect mutations in the $r p o B$ gene associated with rifampicin resistance and mutations in the katG gene and the inh $A$ gene associated with isoniazid resistance.

\section{Coverage}

Based on the tested samples from the period 2003 to 2011, we defined the coverage of molecular fingerprinting of MDR-/XDR-TB as the percentage of MDR-/ XDR-TB isolates included in the molecular surveillance project among the total number of MDR-/XDR-TB cases officially reported to the ECDC for the same period. The ECDC published the surveillance results in The European Surveillance System (TESSy) and in the annual surveillance reports.

\section{Clustering}

A European cluster was defined as two or more MDR-/ XDR-TB strains with identical 24-locus VNTR typing patterns, isolated in at least two different countries.
Results for 15 -locus VNTR typing and VNTR patterns for which one or more loci were missing were also included in the cluster analysis.

\section{Beijing genotype identification}

The Beijing genotype was identified by the specific Beijing branch of the dendrogram with a similarity percentage of 24 -locus VNTR typing of at least $60 \%$. The Beijing branch was determined by 656 isolates confirmed as the Beijing genotype based on spoligotyping. The non-Beijing branches were confirmed as such by spoligotyping of 201 isolates.

\section{Results}

\section{Coverage}

The countries participating in the project reported 16,858 MDR-/XDR-TB cases to the ECDC for the period 2003 to 2011. The total number of MDR-/XDR-TB isolates collected in that period for which VNTR typing data were available amounted to 2,055. Therefore, the coverage of the molecular surveillance for the period 2003 to 2011 was $12 \%$. Six countries reported no molecular typing results at all; excluding these countries, the coverage was $20 \%$. The coverage differed significantly by country and year (Table 1 ).

\section{Typing}

We collected 2,092 VNTR patterns, originating from 2,055 MDR-/XDR-TB patients sampled between 2003 and 2011 in 24 different countries (Figure 1). There were more VNTR patterns than isolates because double alleles were detected in the VNTR patterns of 37

\section{FIGURE 1}

Number of VNTR patterns of multi- and extensively drugresistant Mycobacterium tuberculosis isolates included in the molecular surveillance project, by country of isolation, sampled 2003-2011 (n=2,092)



VNTR: variable number of tandem repeat. 


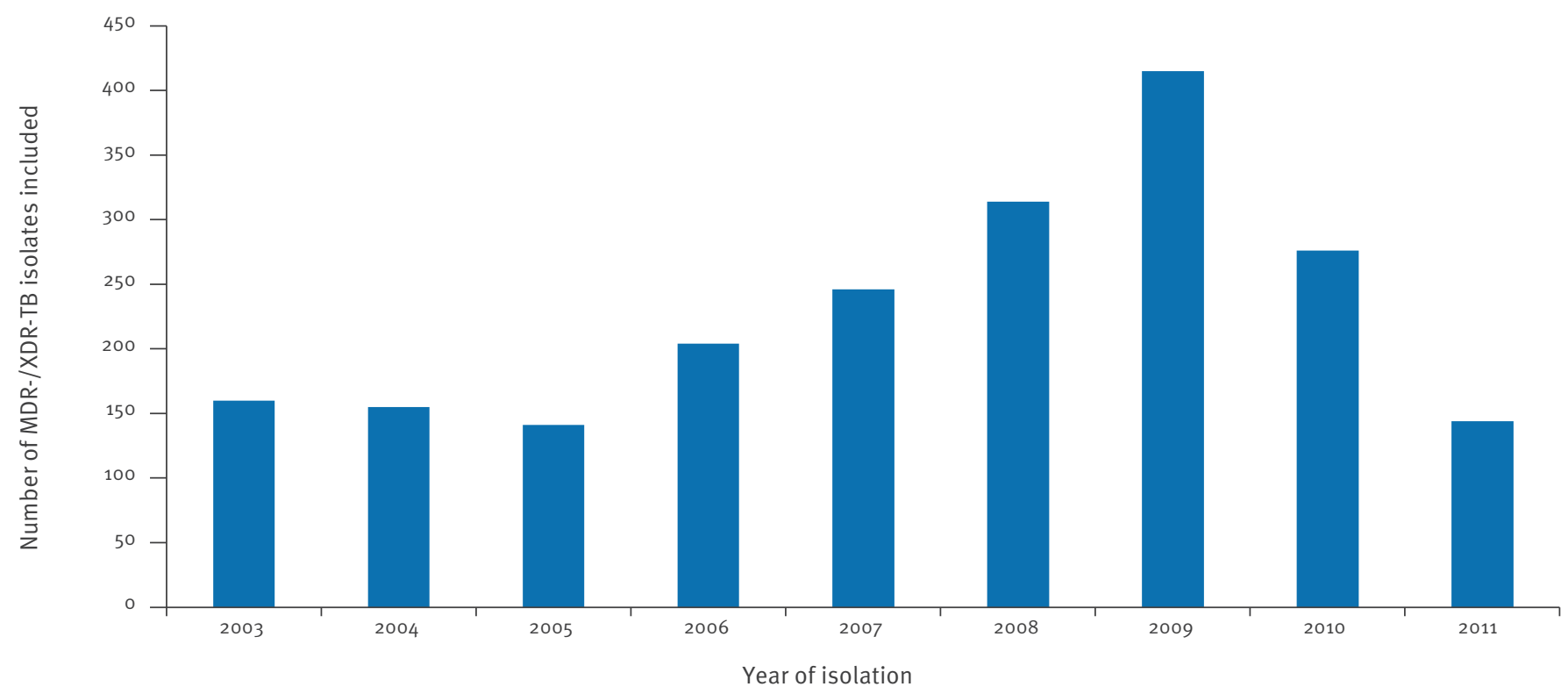

MDR-/XDR-TB: multi- and extensively drug-resistant tuberculosis.

isolates that were included in the project database as separate patterns. For $53 \%(n=1,093)$ of the included isolates, the typing results were produced by the reference laboratory of the country of isolation, and for $47 \%(n=962)$ the molecular typing was performed at the RIVM.

The number of isolates included per year is depicted in Figure 2; 2009 was the year with the highest number of isolates included $(n=415)$. The sex was known for $69 \%$ $(n=1,428)$ of the cases whose isolates were typed: $70 \%$ $(n=999)$ of the MDR-/XDR-TB cases were male and $30 \%$ $(n=429)$ female. The age at the time of TB diagnosis was available for $68 \%(n=1,402)$ of the MDR-/XDR-TB cases included in this study: their mean age was 40 years (range: $1-88$ years).

\section{Clustering}

Comparison of the 2,092 VNTR patterns included in the project resulted in the detection of 79 European clusters. The cluster sizes varied from two to 470 cases per cluster (Figure 3). In total, $45 \%(n=941)$ of all the collected VNTR patterns were part of a European cluster. The geographic composition of these molecular clusters ranged from two to 17 countries.

For $73 \%(n=691)$ of the European clustered cases, the country of origin of the patient was known. In total $73 \%$ $(n=505)$ of these patients were resident in the country of isolation and $27 \%(n=186)$ originated from abroad. Excluding all clustered cases from Estonia $(n=490$ for which the country of origin was known) because of the overrepresentation of samples from Estonia, the distribution was $44 \%(n=89)$ and $56 \%(n=112)$, respectively, for the 201 samples for which country of origin was known.

The percentage of samples assigned to a European MDR-/XDR-TB cluster, for the countries which submitted at least 10 isolates to the project database, varied from o to $87 \%$ by country. Clustering on national level was also analysed in this study and varied from o to $92 \%$ by country (Figure 4 ).

A number of the VNTR typing patterns $(n=465 ; 22 \%)$ did not cover all of the 24 loci due to technical problems or because these loci were not tested in the participating laboratories. In total 60 samples with incomplete VNTR patterns were part of molecular clusters (among them 32 samples of the Beijing genotype): $48 \%(n=29)$ of the samples with incomplete VNTR patterns were part of 22 European clusters, while $52 \%(n=31)$ of them belonged to European clusters which had already been defined on the basis of 24-locus VNTR results from at least two other samples from two different countries.

Of all clustered isolates included in the project database, $60 \%(n=470)$ were part of one large VNTR typing cluster (Figure 3; Table 2). This molecular cluster, comprising a VNTR pattern with a Beijing genotype signature, has so far been detected in 17 EU countries. The majority of cases that belonged to this cluster were detected in the Baltic States, mainly in Estonia (Figure 5). Because of the high coverage of reported cases in Estonia, 98\% for the period 2003 to 2009, the growth dynamics of this largest molecular cluster are depicted 


\section{FIGURE 3}

Size of European clusters of multi- and extensively drug-resistant tuberculosis cases detected in the molecular surveillance project, 2003-2011 ( $\mathrm{n}=79$ clusters)

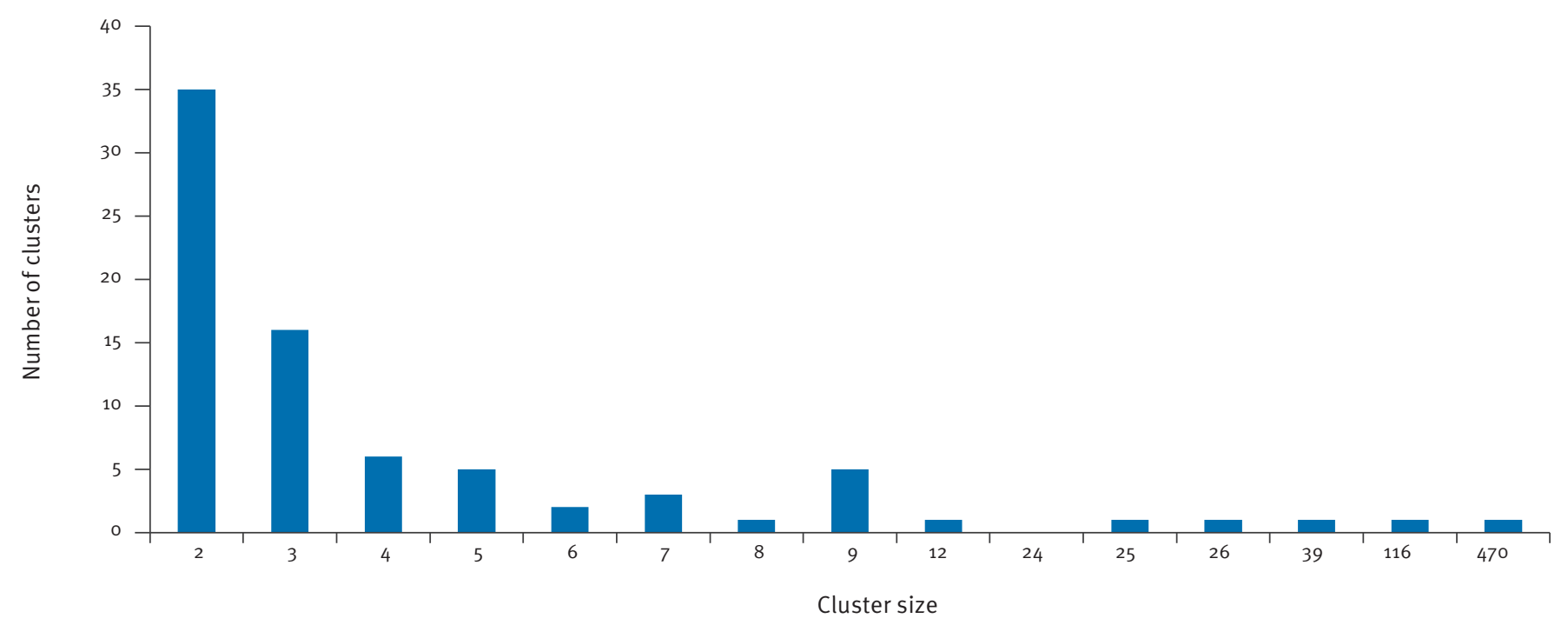

in Figure 6. In 2009, 72 isolates in the cluster originated from Estonia; in the following years, this number decreased to $42-55$ isolates per year.

For a selection of $48(10 \%)$ isolates in the largest molecular cluster, isolated in different countries and years, we determined the mutations underlying the resistance mechanism. All but one of the tested MDR-/ XDR-TB isolates in the VNTR cluster with Beijing genotype revealed the same combination of mutations associated with rifampicin and isoniazid resistance: rров S531L and katG S315T. One exceptional MDR-/ XDR-TB isolate harboured the $r p o B \mathrm{H}_{526} \mathrm{Y}$ and $\mathrm{katG}$
S315T mutations. For 39 of these 48 strains, the resistance to fluoroquinolones and the injectable drugs was tested phenotypically: 12 were resistant to both, five only to fluoroquinolones, 12 only to injectable drugs, and 10 showed no resistance.

\section{Characteristics of clustered MDR-/XDR-TB cases}

Sex and age did not differ between clustered and nonclustered cases. The overall mean age was 40 years (range: 1-88 years). The percentage of VNTR patterns who were part of a European cluster was $54 \%(n=548)$

\section{FIGURE 4}

Percentage of cases in European $(n=941)$ and national $(n=1,086)$ clusters of multi- and extensively drug-resistant tuberculosis, by country, molecular surveillance project, 2003-2011

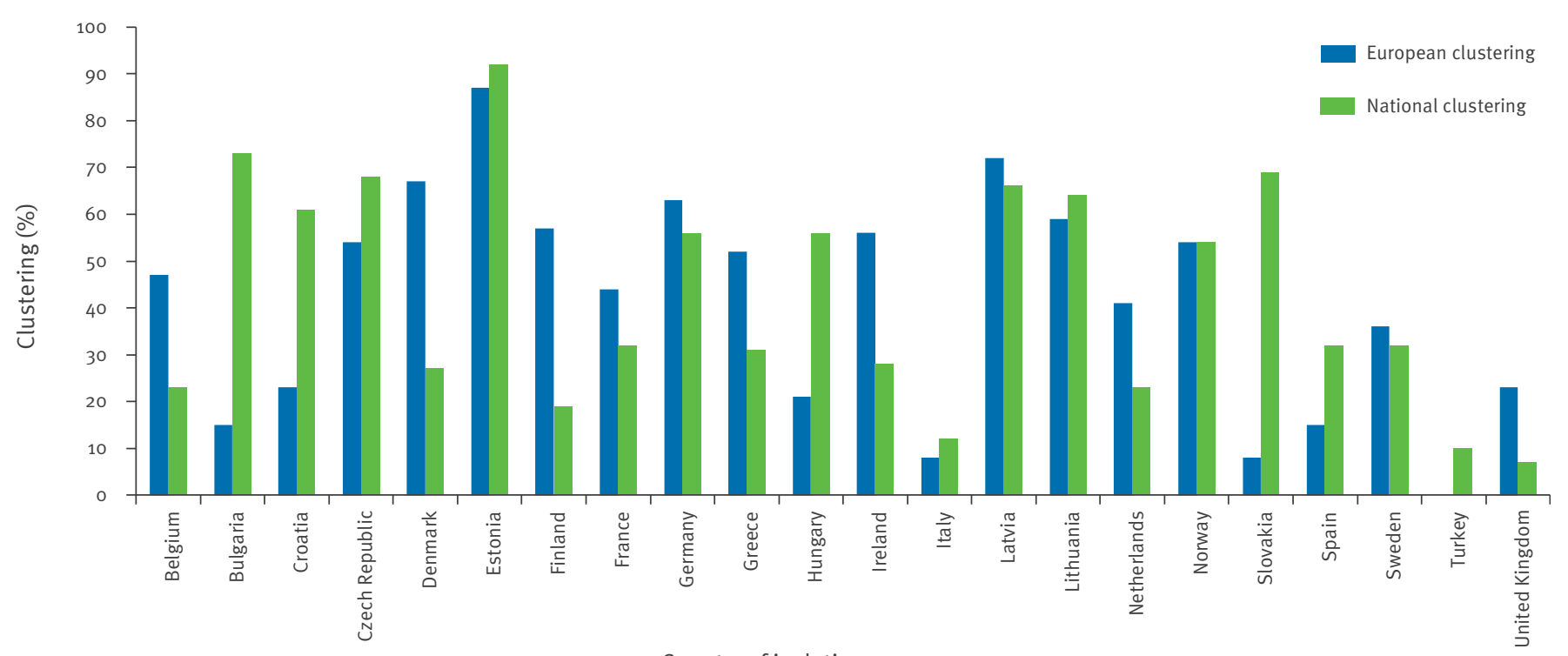

Country of isolation 
24-locus VNTR pattern of the largest multi- and extensively drug-resistant tuberculosis cluster with a Beijing genotype signature detected in the European Union, 2003-2011 ( $n=470$ isolates)

\begin{tabular}{|c|c|c|c|c|c|c|c|c|c|c|c|c|c|c|c|c|c|c|c|c|c|c|c|c|}
\hline Genome position number & $\begin{array}{l}0 \\
\infty \\
i n\end{array}$ & $\begin{array}{l}0 \\
\text { ลे } \\
\text { ㅎ }\end{array}$ & $\begin{array}{l}\text { N } \\
\infty \\
\infty\end{array}$ & $\begin{array}{l}\text { ஜ } \\
\text { ด̆ }\end{array}$ & 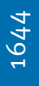 & $\begin{array}{l}N \\
\vec{m} \\
\vec{m}\end{array}$ & $\stackrel{\stackrel{+}{+}}{\stackrel{+}{*}}$ & $\hat{\hat{n}}$ & $\begin{array}{l}\stackrel{n}{o} \\
\vec{N}\end{array}$ & $\stackrel{\vec{~}}{\stackrel{+}{N}}$ & $\begin{array}{l}\circ \\
\text { a } \\
\text { m }\end{array}$ & $\begin{array}{l}0 \\
\stackrel{ }{\stackrel{n}{*}} \\
\stackrel{+}{*}\end{array}$ &  & $\begin{array}{l}\stackrel{n}{\sim} \\
\stackrel{-}{r}\end{array}$ & $\begin{array}{l}\text { N } \\
\stackrel{0}{+} \\
\forall\end{array}$ & $\stackrel{+}{\sim}$ & $\stackrel{\vec{m}}{\stackrel{N}{N}}$ & $\stackrel{\infty}{\underset{m}{+}}$ & 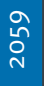 & 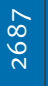 & $\begin{array}{l}\hat{\circ} \\
\text { ○े }\end{array}$ & $\stackrel{\text { \} }{N}} &{\text { 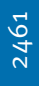 }} &{\stackrel{\vec{n}}{\vec{m}}} \\
{\hline \text { Number of tandem repeats }} &{2} &{7} &{3} &{3} &{3} &{5} &{4} &{4} &{4} &{4} &{3} &{2} &{6} &{5} &{7} &{2} &{5} &{3} &{2} &{1} &{3} &{4} &{2} &{3} \\
$\hline
\end{tabular}

VNTR: variable number of tandem repeat.

for isolates from male cases and $49 \%(n=213)$ for isolates from female cases.

Forty-four per cent $(n=920)$ of the analysed VNTR patterns of MDR-/XDR-TB isolates were assigned to the Beijing genotype with a similarity of at least $74 \%$ on the basis of 24 -locus VNTR typing. For $71 \%(n=656)$ of the 920 isolates, the Beijing genotype was confirmed by RFLP typing and/or spoligotyping and a non-Beijing genotype was confirmed for $17 \%(n=201)$ of the 1,173 strains identified as non-Beijing.

In total, $77 \%$ ( $n=726$ ) of the clustered cases were caused by Beijing genotype strains with 37 different VNTR patterns (the two largest molecular clusters were caused by Beijing genotype strains). Among non-clustered cases, $17 \%(n=194)$ were caused by Beijing strains ( $p<0.05$ ). The mean age for MDR-/XDR-TB cases caused by Beijing genotype strains was not different from that of non-Beijing MDR-/XDR-TB cases: 41.9 vs 39.5 years. In relation to the sex distribution, the Beijing genotype was more often detected in male than in female patients: $53 \%(n=539)$ vs $47 \%(n=206)$.

The susceptibility of the $M$. tuberculosis strains to second-line drugs was known for $53 \%(n=1,080)$ of the isolates. Twelve per cent $(n=132)$ of them were XDR-TB, and 135 VNTR patterns were found for them. There were significantly more men than women among XDR-TB patients: $69 \%(n=91)$ vs $23 \%(n=31)(p<0.05)$. XDR-TB was significantly more often detected in MDR-TB strains of the Beijing genotype than in MDR-TB

\section{FIGURE 5}

Geographical distribution of cases in the largest European multi- and extensively drug-resistant tuberculosis cluster 2003$2011(n=470)$

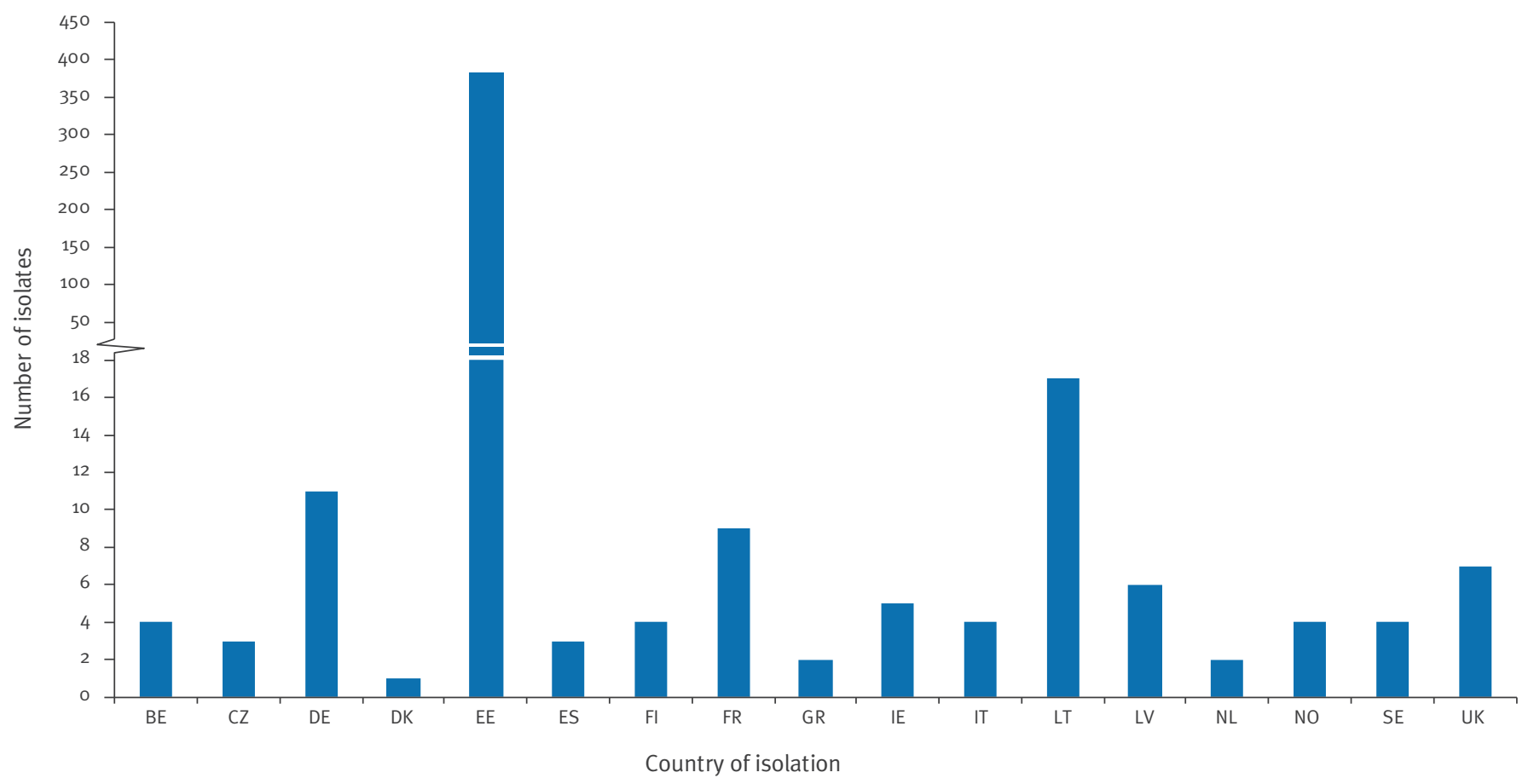

BE: Belgium; CZ: Czech Republic; DE: Germany; DK: Denmark; EE: Estonia; ES: Spain; FI: Finland; FR: France; GR: Greece; IE: Ireland; IT: Italy; LT: Lithuania; LV: Latvia; NL: the Netherlands; NO: Norway; SE: Sweden; UK: United Kingdom. 
Multi- and extensively drug-resistant Mycobacterium tuberculosis isolates belonging to the largest European cluster, by year, Estonia, 2003-2009 ( $\mathrm{n}=384)$

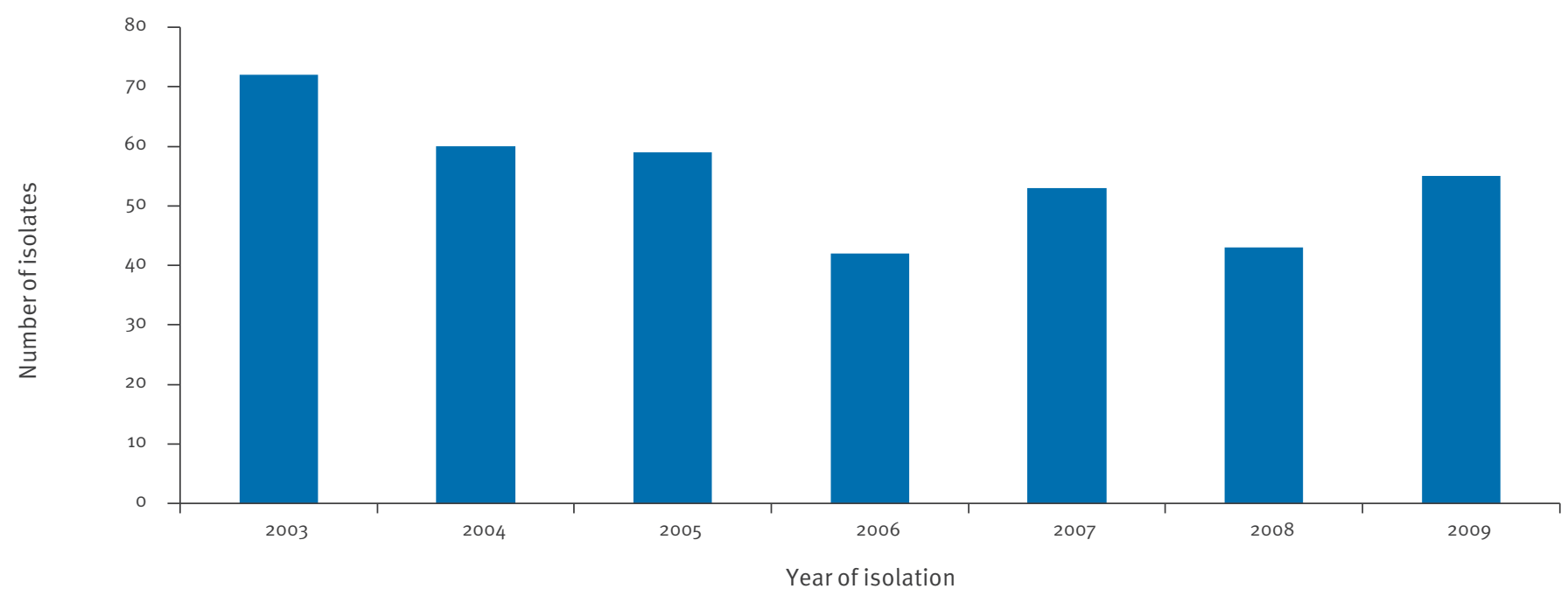

strains of non-Beijing genotypes: $86 \%(n=116)$ vs $14 \%$ $(n=19)(p<0.01)$. In addition, $78 \%(n=105)$ of the XDR-TB VNTR patterns belonged to eight international clusters; six of these clusters were determined as the Beijing genotype.

\section{Discussion}

Almost half of the VNTR patterns collected in this molecular surveillance study of MDR/XDR M. tuberculosis was assigned to international European clusters, and $60 \%$ of these were part of a single, large European cluster. This molecular cluster, associated with the spread of a Beijing genotype strain, has so far been detected in 17 European countries. It was previously described in the EU by RFLP typing and notified for the first time in $2003[1,2]$. The RFLP typing results were available for $63 \%(n=125)$ of the isolates obtained from the largest VNTR cluster in 2003 to 2005. This confirmed the clustering of these cases on the basis of both RFLP and VNTR typing. Overall, the Beijing genotype was significantly associated with clustering, and therefore with possible (international) transmission and spread.

The high proportion of molecular clustering (45\%) in the EU suggests that MDR-/XDR-TB cases may be transmitted and not acquired. The lack of coverage and the wide variation in the number and time period of collected samples submitted by the participating countries, however, reduce the representativeness of this observation. Furthermore, a high proportion of the European clustered cases (73\%) were patients originating from the country of isolation rather than immigrants. Even when excluding all Estonian isolates, the percentage was still $44 \%$. This confirms that MDR-/ XDR-TB transmission was taking place and that not all detected molecular clusters were a result of human migration.
The high percentage of European and national clustering, especially in Estonia (87\% and 92\%) and Latvia ( $72 \%$ and $66 \%$ ), indicates that transmission has been ongoing in this region for a prolonged period [7], and this calls into question the infection control practices and the quality of treatment. In contrast, the low percentage of clustering in Italy (8\%) and Spain (15\%) indicates that the MDR-TB problem in these regions is mainly due to TB imported by immigration from countries not participating in the project, as suggested earlier $[8,9]$. In addition, countries with a higher percentage of European clustering compared to the percentage of national clustering, e.g. the Netherlands ( $41 \%$ vs $23 \%$ ) and Finland ( $57 \%$ vs $19 \%$ ), are examples of importation of MDR-/XDR-TB from European countries and a health system that prevents national transmission.

XDR-TB was detected in $12 \%(n=132)$ of the $M$. tuberculosis isolates for which second-line drug susceptibility data was available. This is slightly higher than described earlier for the MDR-TB cases examined in the period 2006 to 2009 [2]. The Beijing genotype is associated with multidrug resistance in many settings [10]. In this European surveillance project, the Beijing genotype was significantly associated with XDR-TB, in contrast to strains of non-Beijing genotypes: respectively $86 \%(n=116)$ and $14 \%(n=19)$. The association of the Beijing genotype with resistance has been studied extensively; potential underlying mechanisms include a higher mutation frequency of the rpoB gene in strains of the Beijing genotype, resulting in a higher ability to withstand rifampicin exposure [11].

The most important limitation of our study is the poor coverage and thus the possible selection bias; the percentage of MDR/XDR M. tuberculosis isolates that were actually submitted by the participating countries in the period from 2003 to 2011 ranged from $0 \%$ to more than $100 \%$. Limited coverage also affected the timeliness 
of delivery of data. Several countries, including a few large ones, reported limited data, although it was agreed in the project to send real-time typing results. The effect of this limitation is a possible underestimation of international transmission of MDR-/XDR-TB in the EU. An important implication of our study is that especially in western EU countries, the percentage of clustered MDR-/XDR-TB cases is low. This implies that resistance was either acquired in the patient in the country where the strain was isolated, or a consequence of sequential import of unrelated cases from endemic regions.

In contrast, in the eastern EU countries and especially the Baltic States, a large proportion of MDR-/XDR-TB isolates belonged to molecular clusters. Moreover, one large molecular cluster of 470 cases was caused by Beijing strains with identical 24-locus VNTR typing patterns. This implies major and ongoing transmission of an easily transmissible and virulent strain or clone. Forty-seven of the 48 tested isolates in the largest molecular cluster had the same combination of rpoB S $531 \mathrm{~L}$ and katG $\mathrm{S}_{315} \mathrm{~T}$ mutations, associated with rifampicin and isoniazid resistance. There is bacteriological and epidemiological data demonstrating that these mutations result in the lowest loss of fitness in isoniazid- and rifampicin-resistant bacteria $[12,13]$. Resistance to second-line drugs was high variable. The largest international cluster may therefore be caused by one successful MDR-/XDR-TB strain that is responsible for many transmissions, with resistance to secondline drugs developing further in the affected patients. Alternatively, we may be observing the spread of genetically highly similar strains of the Beijing genotype. By whole-genome sequencing, the true percentage of similarity can be determined, and this will help to answer this question.

Another important limitation in this study was the lack of epidemiological data to confirm chains of human transmission. Although the typing data are highly suggestive of spread of successful strains, this still needs to be confirmed.

For this project, we selected VNTR typing as the standard method. This technique was previously shown to be highly reproducible, both within [14] and between laboratories [15]. However, the participants of the ECDC/ RIVM project used a large variation in protocols and methodologies and had different levels of experience in performing VNTR typing. Therefore, we performed two proficiency studies; initial results were disappointing regarding both the intra- and inter-laboratory reproducibility [5]. Although several suggestions for improvements were communicated to participants, this lack in quality may still have influenced the results of the current study, leading to an underestimation of clustering cases. After implementation of several improvements in the methodology and a higher degree of standardisation, the second international proficiency study in 2010 on VNTR typing yielded much better results [16].
In conclusion, large-scale international transmission of MDR-/XDR-TB occurs within the EU and demands increased surveillance and public health action. The $M$. tuberculosis strains with Beijing genotype are large drivers of this international transmission and are associated with the emergence and spread of XDR-TB.

Participants of the ECDC MDR-TB molecular surveillance project:

Ilona Zemanova: The National Institute of Public Health, Prague, Czech Republic;

D. Pieridou-Bagkatzouni: Nicosia General Hospital, Microbiology, Strovolos, Cyprus;

Anna Agejeva: Tartu University Clinics, Department of Mycobacteriology, Tartu, Estonia;

Panayotis Ioannidis: National Reference Laboratory for Mycobacteria, "Sotiria" Chest Diseases Hospital, Athens, Greece;

Tamas Herczeg: Corden International Ltd, National Mycobacteriology Reference Laboratory, Budapest, Hungary; Ulf R. Dahle: Norwegian Institute of Public Health, Oslo, Norway;

Monika Polanová: National Reference Laboratory for Mycobacteria, Nitra, Slovakia;

Elizabeta Bachiyska: National Center of Infectious and Parasitic Diseases, Sofia, Bulgaria;

Urska Bidovec-Stojkovic: Laboratory for Mycobacteria, University Clinic of Respiratory and Allergic Diseases, Golnik, Slovenia;

Timothy Brown: United Kingdom Health Protection Agency, London, United Kingdom;

Daniela M. Cirillo: Reference Laboratory for Tuberculosis, Emerging Bacterial Pathogens Unit, Milan, Italy;

Maryse Fauville-Dufaux: Tuberculosis and Mycobacteria, Scientific Institute of Public Health, Brussels, Belgium;

Margaret M. Fitzgibbon: Irish Mycobacteria Reference Laboratory, Central Pathology Laboratory, Dublin, Ireland;

Ramona Groenheit: Swedish Institute for Communicable Disease Control, Solna, Sweden;

Marjo Haanperä: Mycobacterial Reference Laboratory, National Institute for Health and Welfare, Turku, Finland;

Stefan Niemann: Molecular Mycobacteriology, Forschungszentrum Borstel, Borstel, Germany;

Mihaela Obrovac: National Mycobacteria Reference Laboratory, Croatian National Institute of Public Health, Zagreb, Croatia;

Erik M. Rasmussen: International Reference Laboratory of Mycobacteriology, Copenhagen, Denmark;

Sofia Samper: Servicio de Microbiología, Hospital Universitario Miguel Servet, IIS Aragon, Zaragoza and CIBER Enfermedades Respiratorias, Spain; 
Wladimir Sougakoff: National Reference Centre for Mycobacteria (CNR-MyRMA), Laboratory of Bacteriology, APHP, Paris, France;

Dick van Soolingen: National Tuberculosis Reference Laboratory, National Institute for Public Health and the Environment (RIVM), Bilthoven, The Netherlands;

Petras Stakenas: Institute of Biotechnology, Vilnius University, Lithuania.

\section{Acknowledgements}

Data from the European Union and European Economic Area Member States in this study is part of the ECDC MDR-TB molecular surveillance project. The project is coordinated by ECDC and the implementation was outsourced to the RIVM under ECDC service contract ECDC/08/019 (February 2009February 2012).

Edita Pimkina from Affiliate of Vilnius University Hospital Santariskiu Klinikos, Vilnius University, Lithuania is ac knowledged for providing VNTR patterns.

\section{Conflict of interest}

None declared.

\section{Authors' contributions}

The project participants all contributed significantly to the results of this study.

\section{References}

1. Devaux I, Kremer K, Heersma H, Van Soolingen D. Clusters of multidrug-resistant Mycobacterium tuberculosis cases, Europe. Emerg Infect Dis. 2009;15(7):1052-60. http://dx.doi. org/10.3201/eid1507.080994

2. Devaux I, Manissero D, Fernandez de la Hoz K, Kremer $\mathrm{K}$, van Soolingen D. Surveillance of extensively drugresistant tuberculosis in Europe, 2003-2007. Euro Surveill 2010;15(11): pii=19518.

3. Supply P, Allix C, Lesjean S, Cardoso-Oelemann M, RuschGerdes S, Willery E, et al. Proposal for standardization of optimized mycobacterial interspersed repetitive unit-variablenumber tandem repeat typing of Mycobacterium tuberculosis. J Clin Microbiol. 2006;44(12):4498-510. http://dx.doi. org/10.1128/JCM.01392-06

4. de Beer JL, van Ingen J, de Vries G, Erkens C, Sebek M, Mulder A, et al. Comparative study of IS6110 RFLP and VNTR typing of Mycobacterium tuberculosis in the Netherlands, based on a five year nationwide survey. J Clin Microbiol. 2013:51(4):1193-8. http://dx.doi.org/10.1128/JCM.03061-12

5. de Beer JL, Kremer K, Kodmon C, Supply P, van Soolingen D. First worldwide proficiency study on variable-number tandem repeat typing of Mycobacterium tuberculosis complex strains. J Clin Microbiol. 2012;50(3):662-9. http://dx.doi.org/10.1128/ JCM.00607-11

6. Hillemann D, Rusch-Gerdes S, Richter E. Evaluation of the GenoType MTBDRplus assay for rifampin and isoniazid susceptibility testing of Mycobacterium tuberculosis strains and clinical specimens. J Clin Microbiol. 2007;45(8):2635-40. http://dx.doi.org/10.1128/JCM.00521-07

7. Kruuner A, Hoffner SE, Sillastu H, Danilovits M, Levina K, Svenson SB, et al. Spread of drug-resistant pulmonary tuberculosis in Estonia. J Clin Microbiol. 2001;39(9):3339-45. http://dx.doi.org/10.1128/JCM.39.9.3339-3345.2001

8. Franzetti F, Codecasa L, Matteelli A, Degli Esposti A, Bandera A, Lacchini C, et al. Genotyping analyses of tuberculosis transmission among immigrant residents in Italy. Clin Microbiol Infect. 2010;16(8):1149-54. http://dx.doi. org/10.1111/j.1469-0691.2009.03080.x

9. Gavin P, Iglesias MJ, Jimenez MS, Rodriguez-Valin E, Ibarz $\mathrm{D}$, Lezcano MA, et al. Long-term molecular surveillance of multidrug-resistant tuberculosis in Spain. Infect Genet Evol. 2012;12(4):701-10. http://dx.doi.org/10.1016/j. meegid.2011.05.016

10. Parwati I, van Crevel R, van Soolingen D. Possible underlying mechanisms for successful emergence of the Mycobacterium tuberculosis Beijing genotype strains. Lancet Infect Dis. 2010;10(2):103-11. http://dx.doi.org/10.1016/ S1473-3099(09)70330-5

11. de Steenwinkel JE, Ten Kate MT, de Knegt GJ, Kremer K, Aarnoutse RE, Boeree MJ, et al. Drug Susceptibility of Mycobacterium tuberculosis Beijing Genotype and Association with MDR TB. Emerg Infect Dis. 2012;18(4):660-3. http:// dx.doi.org/10.3201/eid1804.110912

12. Gagneux S, Long CD, Small PM, Van T, Schoolnik GK, Bohannan BJ. The competitive cost of antibiotic resistance in Mycobacterium tuberculosis. Science. 2006;312(5782):1944-6. http://dx.doi.org/10.1126/science.1124410

13. van Doorn HR, de Haas PE, Kremer K, Vandenbroucke-Grauls CM, Borgdorff MW, van Soolingen D. Public health impact of isoniazid-resistant Mycobacterium tuberculosis strains with a mutation at amino-acid position 315 of katG: a decade of experience in The Netherlands. Clin Microbiol Infect. 2006;12(8):769-75.

14. Supply P, Lesjean S, Savine E, Kremer K, van Soolingen D, Locht C. Automated high-throughput genotyping for study of global epidemiology of Mycobacterium tuberculosis based on mycobacterial interspersed repetitive units. J Clin Microbiol. 2001;39(10):3563-71. http://dx.doi.org/10.1128/ JCM.39.10.3563-3571.2001

15. Cowan LS, Mosher L, Diem L, Massey JP, Crawford IT. Variable-number tandem repeat typing of Mycobacterium tuberculosis isolates with low copy numbers of IS6110 by using mycobacterial interspersed repetitive units. J Clin Microbiol. 2002;40(5):1592-602. http://dx.doi.org/10.1128/JCM.40.5.1592-1602.2002

16. de Beer JL, Ködmön C, van Ingen J, Supply P, van Soolingen D. Second worldwide proficiency study on variable number of tandem repeats typing of Mycobacterium tuberculosis complex. Int J Tuberc Lung Dis. 2014. Forthcoming. 